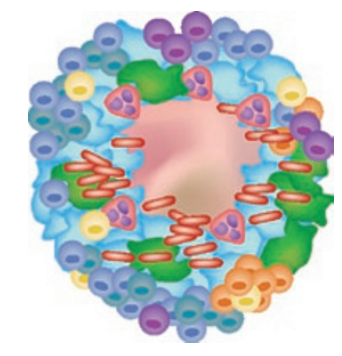

p 271

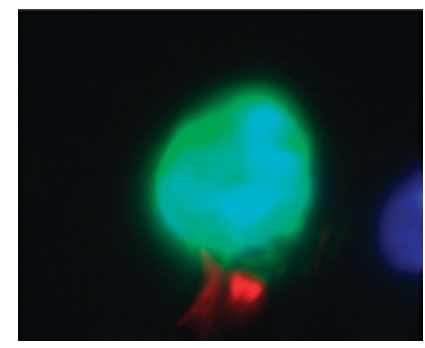

p 279

\title{
Focus on the immunology of tuberculosis
}

Mycobacterium tuberculosis infects nearly one-third of the world's population and is a major killer. This issue of Mucosal Immunology, graciously guest-edited by Dr. Alan Sher, is focused on major new developments in our understanding of the immunology of tuberculosis, with the goal of stimulating thought on new mechanisms of prevention and treatment of this devastating disease.

\section{Mucosal infections}

Sharon Perry and colleagues discuss data indicating that distinct mucosal infections influence the acquisition and outcome of $M$. tuberculosis. See page 246

\section{Innate cytokines}

In this insightful review, Andrea Cooper and colleagues describe major innate cytokine pathways in the establishment and maintenance of

M. tuberculosis. See page 252

\section{Interleukin-10}

Paul Redford and colleagues examine the central role of interleukin-10 in the fine balance of immunopathology and protection against M. tuberculosis. See page 261

\section{Granulomatous inflammation}

JoAnne Flynn and colleagues discuss the key role of the macrophage in $M$. tuberculosis granulomas.

See page 271

\section{Macrophage apoptosis}

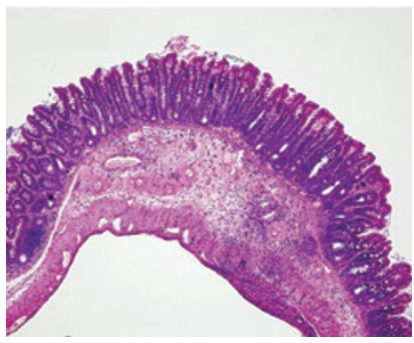

p 294
Samuel Behar and colleagues explain how M. tuberculosis can subvert macrophage apoptosis, an important innate defense mechanism. See page 279

\section{T-cell control of tuberculosis}

Kevin Urdahl and colleagues discuss the induction and control of T-cell responses against

M. tuberculosis infection. See page 288

\section{Leptin and E. histolytica}

Xiaoti Guo and colleagues have identified an essential role for leptin signaling in resistance to Entamoeba histolytica infection. See page 294

\section{PPAR- $\gamma$ and macrophages}

Raquel Hontecillas and colleagues show that peroxisome proliferator-activated receptor- $\gamma$ regulates macrophage function and T-cell responses in experimental colitis. See page 304

\section{Ndfip1 in Th2 inflammation}

Hilda Ramon and colleagues indicate a role for the adaptor protein in intestinal inflammation.

See page 314

\section{Lymphocyte control of Nod2}

Claudia Duerr and colleagues demonstrate that intestinal lymphocytes can degrade muramyl dipeptide and prevent its recognition by nucleotide-binding oligomerization domain 2. See page 325

\section{Epithelial defense}

Charles Wira and colleagues demonstrate that epithelial cell secretions from the upper female reproductive tract can inhibit the growth of Neisseria gonorrhoeae, Candida species, and HIV.

See page 335

\section{DCs in lung allergy}

Alejandro Ortiz-Stern and colleagues show a essential role for langerin-positive dendritic cells in lipopolysaccharide-induced exacerbation of airway inflammation. See page 343

\section{Muc2 mutations and IBD}

Rajaraman Eri and colleagues demonstrate that missense mutations in Muc2 result in endoplasmic reticulum stress in goblet cells and spontaneous intestinal inflammation. See page 354 